\title{
Smartphone Homecare Monitoring of Hearts
}

\author{
Harold Szu, ${ }^{1}$ Charles Hsu, ${ }^{2}$ Gyu Moon, ${ }^{3}$ Joseph Landa, ${ }^{4}$ \\ Hiroshi Nakajima, ${ }^{5}$ and Yutaka Hata ${ }^{6}$ \\ ${ }^{1}$ Department of Biomedical Engineering, The Catholic University of America, Washington, DC 20064, USA \\ ${ }^{2}$ Trident Systems Inc., Fairfax, VA 22030, USA \\ ${ }^{3}$ Department of Electronic Engineering, Hallym University, Chunsheon-si, Gangwon-do 200-702, Republic of Korea \\ ${ }^{4}$ BriarTek Incorporated, Alexandria, VA 22035, USA \\ ${ }^{5}$ OMRON Corp., 9-1 Kizugawadai, Kizugawa-city, Kyoto 619-0283, Japan \\ ${ }^{6}$ Department of Electrical Engineering and Computer Science, University of Hyogo, 2167 Shosha Himeji, \\ Hyogo 671-2280, Japan
}

Correspondence should be addressed to Harold Szu; szuharoldh@gmail.com

Received 25 May 2012; Accepted 21 October 2012

Academic Editor: Soo-Young Lee

Copyright (C) 2013 Harold Szu et al. This is an open access article distributed under the Creative Commons Attribution License, which permits unrestricted use, distribution, and reproduction in any medium, provided the original work is properly cited.

\begin{abstract}
Homecare monitoring blood pressures and heartbeats are commercially available using dedicated devices, for example, wrist watch, pulse oximetry. With the advent of Smartphone and compressive sensing technology, we wish to monitor precisely the electrical waveforms of heartbeats called the electrocardiography (ECG) for an aging global villager biomedical wellness homecare system. Our design separates into 3 innovative modules within the size-weight and power-cost bandwidth (Swap-CB) limitation. We develop each separately but in concert with one another: (i) Smart Electrode (adopting a low-power-mixed signal embedded with modern compressive sensing firmware and applying the nanotechnology to improve the electrodes' contact impedance as well as novel transduction mechanism, between ECG and electronics, e.g., a pressure mattress coupling, or fiber-optics coupling); (ii) Learnable Database (utilizing adaptive wavelets transforms for systolic and diastolic P-QRS-T-U features extraction Aided Target Recognition and adopting Sequential Query Language for a relational database allowing distant monitoring and retrievable); (iii) Smartphone (inheriting a large touch screen interface display with powerful computation capability and assisting caretaker reporting system with GPS and ID and two-way interaction with patient panic button for programmable emergence reporting procedure). While (i) is novel, (ii) and (iii) are mature. Together, they can eventually provide a supplementary home screening system for the post- or the prediagnosis care at home with a built-in database searchable with the time, the place, and the degree of urgency happened, using in situ screening.
\end{abstract}

\section{Introduction}

Home-alone senior tends to suffer and worry about midnight and early morning crisis, heart attack, or stroke. While their bodies secrete hormones regulating hearts and blood pressures ready for the wake-up actions, their limbs are still stif $\mathrm{f}$ suffering bad circulations over the sleep. For caretakers, we design an affordable, and comfortable biomedical wellness (BMW) monitoring device of detail electrocardiogram (ECG) waves for early warning monitoring at homes. Thus, we review heart monitoring ECG as a common sense baseline, before we present compressing sensing technology (reader may skip over the following and go directly to Compressive Sensing).

A healthy heart is controlled by a sequence of electrical signals fired in order, following the proper timing with an oxygen-rich red blood taking from our lungs to the Aorta upper chambers called Atrium, and down to the lower chambers called Ventricle and pump the blood with a systolic pressure through the artery out to our body vital organs. The heart also does the sucking up against the gravity, using passive muscular lining and one-way valves generating a diastolic pressure for the replenishment of oxygen-poor bluish blood returning from capillary beds and veins. 


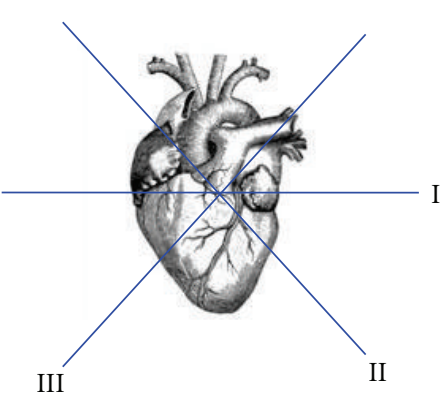

(a)

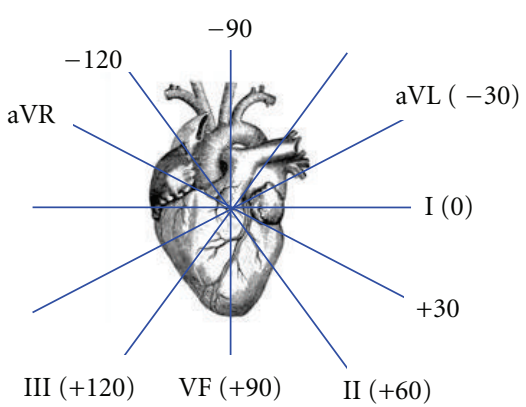

(b)

Figure 1: The first three leads of ECG devised by Einthoven constitute the Einthoven triangle [1]. Lead I is used as the reference lead and is located at 0 degrees. Positive designations increase by 30 degree increments in a clockwise direction to 180 degrees. Lead II appears at 60 degrees. Augmented limb leads (aVL, aVR, aVF) are unipolar limb leads, containing only a positive pole. aVL is designated at -30 degrees, aVF at 90 degrees, and lead aVR at -150 degrees.

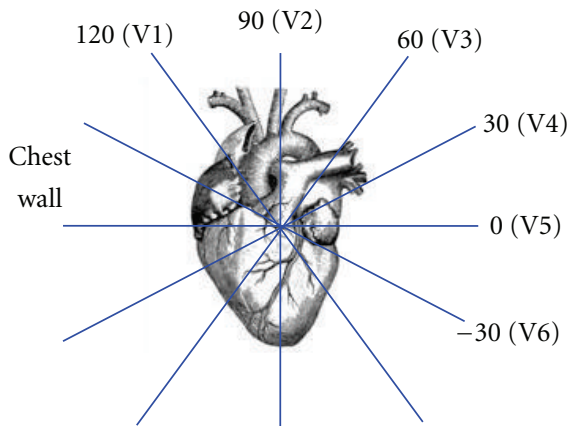

FIGURE 2: By joining the wires from the right arm, left arm, and left foot with 5000 Ohm resistors Frank Wilson defines an "indifferent electrode" later called the "Wilson Central Terminal." The combined lead acts as an earth and is attached to the negative terminal of the ECG. An electrode attached to the positive terminal then becomes "unipolar" and can be placed anywhere on the body. Wilson defines the unipolar limb leads VR, VL, and VF where "V" stands for voltage (the voltage seen at the site of the unipolar electrode).

The basic principle underlying the genesis of the ECG waveforms and their polarity-positive, negative, or isoelectric - is as follows.

(i) Electrical activity moving toward the positive terminal of a lead generates a positive wave.

(ii) Electrical activity moving away from the positive terminal of a lead generates a negative wave.

(iii) Electrical activity moving at 90 degrees to a lead will generate a biphasic wave (equal positive and negative components, or isoelectric).

The first three leads devised by Einthoven constitute the Einthoven triangle. These leads are numbered I, II, and IIIthese are bipolar leads because they contain both a positive and a negative poles shown in Figure 1. Dr. Frank Wilson of University of Michigan [2] introduced 6 precordial leads, relating the transverse plane of the body. V1 is placed in the 4 th intercostal space just to the right of the sternum. V2 placed in the 4th intercostal space to the left of the sternum. $\mathrm{V} 4$ is placed in the 5th intercostal space on the midclavicular line. V3 is then placed halfway along a straight line between $\mathrm{V} 2$ and V3. V5 and V6 are placed lateral to V4 in the anterior axillary and midaxillary lines. The following diagram shows the orientation of the six precordial leads in Figure 2. The test data illustrated in this paper is based on this model.

A typical ECG tracing of the cardiac cycle (heartbeat) consists of a P wave, a QRS complex, a T wave, and a $\mathrm{U}$ wave which is normally visible in 50 to $75 \%$ of ECGs. The baseline voltage of the electrocardiogram is known as the isoelectric line, typically measured as the portion of the tracing following the $\mathrm{T}$ wave and preceding the next $\mathrm{P}$ wave. The waves and the intervals to interpret the electrical activity of the hearts are listed in Table 1, and the structure diagram of the normal human heart from an anterior view is depicted in Figure 3. The healthy ECG waveform is illustrated in Figure 4(a), while the abnormal one in Figure 4(b).

From the practical and experimental studies and research, the abnormal ECG heart beats are categorized as follows:

(i) Ventricle Depolarization (reducing Peak R-Trough S) to $\mathrm{T}$ for Ventricle Repolarization time width;

(ii) heart attacks;

(iii) Atrial Fibrillation (AF) (arrhythmia, or abnormal heartbeat): Sino-Atrial (SA) node at right atrium 
TABLE 1: The waves and the intervals to interpret the electrical activity of the hearts.

\begin{tabular}{|c|c|c|}
\hline Feature & Description & Duration \\
\hline RR interval & The interval between an $\mathrm{R}$ wave and the next $\mathrm{R}$ wave. Normal resting heart rate is between 60 and $100 \mathrm{bpm}$ & 0.6 to $1.2 \mathrm{~s}$ \\
\hline P wave & $\begin{array}{l}\text { During normal atrial depolarization, the main electrical vector is directed from the SA node towards the } \\
\text { AV node and spreads from the right atrium to the left atrium. This turns into the P wave on the ECG }\end{array}$ & $80 \mathrm{~ms}$ \\
\hline PR interval & $\begin{array}{l}\text { The PR interval is measured from the beginning of the P wave to the beginning of the QRS complex. The } \\
\text { PR interval reflects the time the electrical impulse takes to travel from the sinus node through the AV } \\
\text { node and entering the ventricles. The PR interval is therefore a good estimate of AV node function }\end{array}$ & 120 to $200 \mathrm{~ms}$ \\
\hline PR segment & $\begin{array}{l}\text { The PR segment connects the P wave and the QRS complex. The impulse vector is from the AV node to } \\
\text { the bundle of His to the bundle branches and then to the Purkinje Fibers. This electrical activity does not } \\
\text { produce a contraction directly and is merely traveling down towards the ventricles and this shows up flat } \\
\text { on the ECG. The PR interval is more clinically relevant }\end{array}$ & 50 to $120 \mathrm{~ms}$ \\
\hline QRS complex & $\begin{array}{l}\text { The QRS complex reflects the rapid depolarization of the right and left ventricles. They have a large muscle } \\
\text { mass compared to the atria and so the QRS complex usually has much larger amplitude than the P wave }\end{array}$ & 80 to $120 \mathrm{~ms}$ \\
\hline J-point & $\begin{array}{l}\text { The point at which the QRS complex finishes and the ST segment begins. Used to measure the degree of } \\
\text { ST elevation or depression present }\end{array}$ & N/A \\
\hline ST segment & $\begin{array}{l}\text { The ST segment connects the QRS complex and the T wave. The ST segment represents the period when } \\
\text { the ventricles are depolarized. It is isoelectric }\end{array}$ & 80 to $120 \mathrm{~ms}$ \\
\hline T wave & $\begin{array}{l}\text { The T wave represents the repolarization (or recovery) of the ventricles. The interval from the beginning of } \\
\text { the QRS complex to the apex of the T wave is referred to as the absolute refractory period. The last half of } \\
\text { the T wave is referred to as the relative refractory period (or vulnerable period) }\end{array}$ & $160 \mathrm{~ms}$ \\
\hline ST interval & The ST interval is measured from the J point to the end of the T wave & $320 \mathrm{~ms}$ \\
\hline QT interval & $\begin{array}{l}\text { The QT interval is measured from the beginning of the QRS complex to the end of the T wave }(<0.4 \mathrm{sec}= \\
400 \mathrm{~ms}) \text {. A prolonged QT interval is a risk factor for ventricular tachyarrhythmias and sudden death. It } \\
\text { varies with heart rate and for clinical relevance requires a correction for this, giving the QTc }\end{array}$ & 300 to $430 \mathrm{~ms}$ \\
\hline U wave & $\begin{array}{l}\text { The } \mathrm{U} \text { wave is hypothesized to be caused by the repolarization of the interventricular septum. They } \\
\text { normally have a low amplitude, and even more often completely absent. They always follow the T wave } \\
\text { and also follow the same direction in amplitude. If they are too prominent we suspect hypokalemia, } \\
\text { hypercalcemia, or hyperthyroidism usually }\end{array}$ & \\
\hline J wave & $\begin{array}{l}\text { The J wave, elevated J-Point or Osborn Wave, appears as a late delta wave following the QRS or as a small } \\
\text { secondary R wave. It is considered pathognomonic of hypothermia orhypocalcemia }\end{array}$ & \\
\hline
\end{tabular}

triggers the atria pushing blood into the ventricles. Atrio-Ventricular (AV) node contracts the ventricles, pumping blood to the lungs and body;

(iv) abnormalities of the left heart;

(v) abnormalities of the right heart;

(vi) abnormalities of the atria;

(vii) abnormally fast rates (tachycardias);

(viii) abnormally slow rates (bradycardias and conduction blocks).

A number of featured ECG devices can be found in the commercial market. These ECG analyses provide the information on the heart beat rate, rhythm, and the ECG waveform. Selected products are shown in Table 2.

\section{Compressive Sensing ECG}

In 2006, several mathematicians including Candes of CalTech, Romberg of Georgia Institute of Technology, Fields Prize winner Terrence Tao of UCLA, and David Donoho of
Stanford developed a sparse acquisition of image algorithm. Their motivation was to decrease the total acquisition time for MRI imaging by reducing the number of images taken necessarily, yet preserving the original image quality. When the information degree of freedom of number $M \ll N$ which is much smaller than the representation image domain pixel numbers, we allow to do a FFT or DWT to manifest the concentration, then a subsampling on the transformed domain is called the compressive sensing (not postprocessing compression) to make the patients suffering less during the data acquisition. Then, the radiologists spent a good time to recover the high definition image pixel values by solving the ill-posed inverse with trials and errors, a linear programming at the $l_{1}$-norm minimization, under L2 similarity constraint (cf. (3)). By shortening the time, patients will suffer less from (i) radioactive contrast agents (short-lived Gallium isotopes) injected less into the blood stream with an even shorter half live, and (ii) the intense (27 Tesla) magnetic field required by f-MRI systems to clearly see the fine specimen radioactive agents. Figure 5 shows the Logan-Shepp phantom and its least squares reconstruction after Tomography Central Slice Theorem composing 1-D Fourier-sampling projection. 


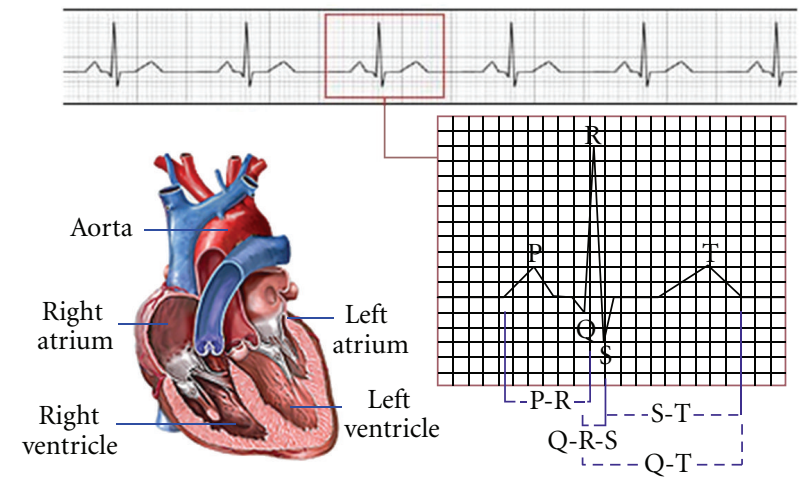

FIGURE 3: Shows the structure diagram of the normal human heart from an anterior view. In the heart, a ventricle is one of two large chambers that collect and expel blood received from an atrium towards the peripheral beds within the body and lungs. The atrium (an adjacent/upper heart chamber that is smaller than a ventricle) primes the pump.

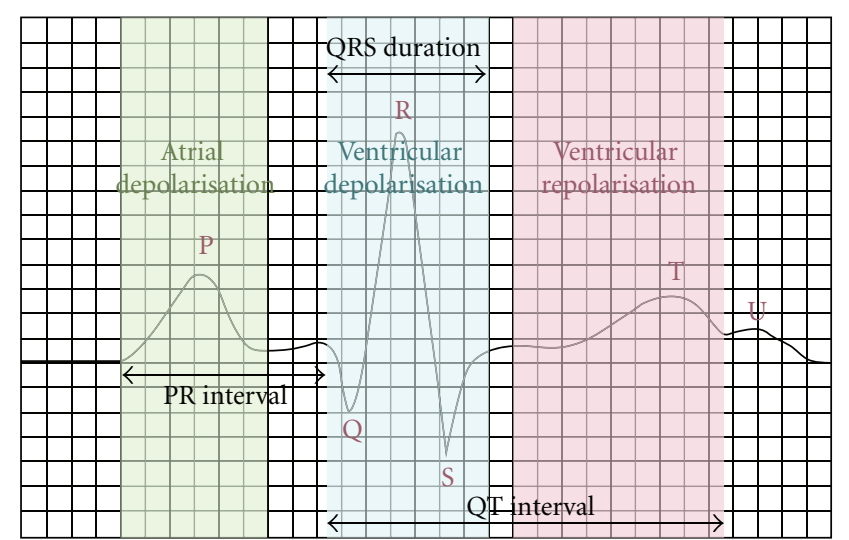

(a)

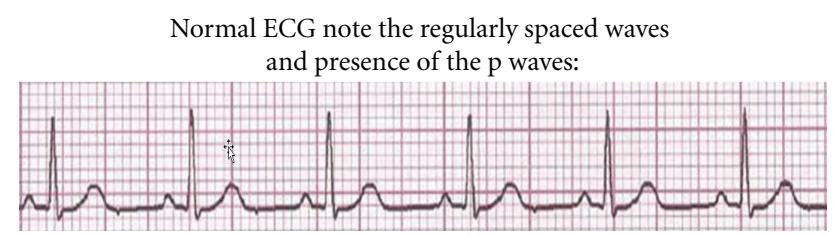

Atrial fibrillation note the irregularly spaced waves and lack of the p waves:

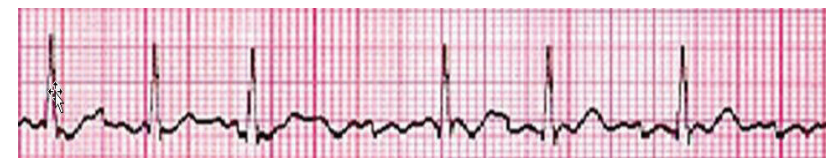

(b)

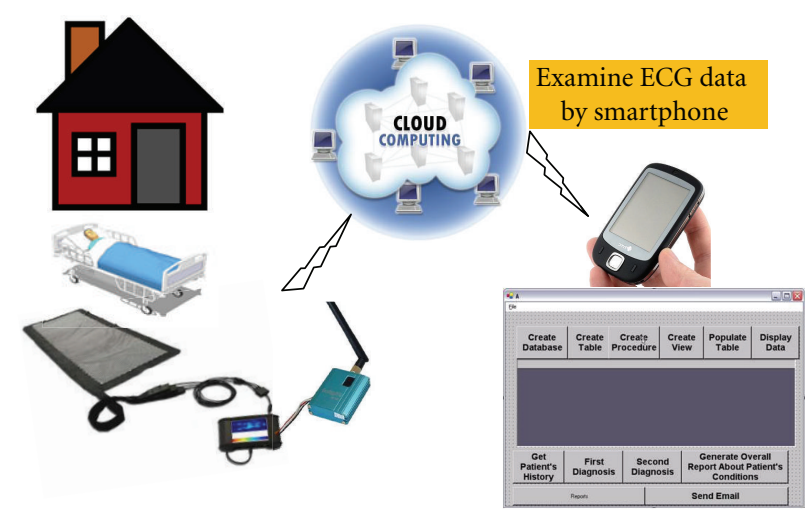

(c)

FIGURE 4: (a) Shows the healthy ECG waveform, (b) abnormal heart beats are exemplified, (c) affordable air mattress ECG home monitoring system.

Their mathematical sampling trick is based on an unstructured, sparse rectangular filtering matrix $\left[\Phi_{i, j}\right]$, which is mainly composed of uniformly random bipolar analog value (representing by threshold many 0's and sparsely and randomly located 1's). Their linear combination sensing matrix $\left[\Phi_{i, j}\right]$ can be visualized as an filter mask; the 1's are translucent holes that pass on pixel values, while the numerous 0's pass no information. The filter decides which parts of the image of size $N$ get sampled. Such sparse rows of 1's among many zeros are pseudoorthogonal to one another, due to a 

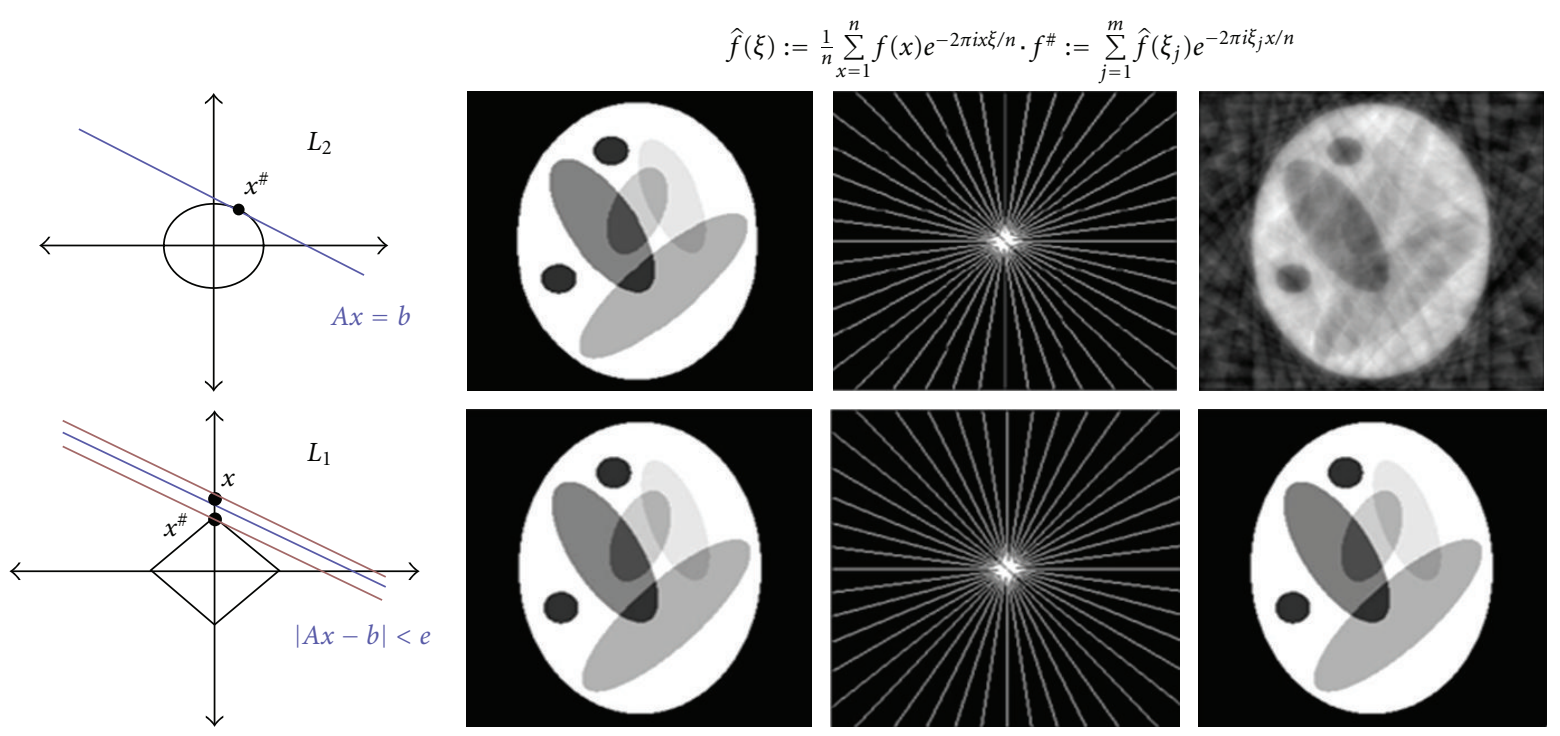

FIgURE 5: Compressive sensing X-ray tomography: the Logan-Shepp phantom and its least squares reconstruction after Tomography Central Lice Theorem composing 1-D Fourier-sampling projection along 22 radial lines (here $m / n \approx 0.05$ ) into 2-D sparse spectrum which are then Fourier inverted to produce a sharp image under the $l_{1}$ norm minimization (bottom right) better than the $l_{2}$ norm (top right). $Y=[\Phi] x+z$, assuming that $z$ has norm less than some error tolerance $e$. MRI Fourier Tomography Reconstruction with 5\% sparse sampling using an energy LMS constraint $L_{2}$ versus magnitude convex hull constraint $L_{1}$ for $M=22$ tomography projection measurements and inverted such an underdeterminant rectangular matrix equation of degree $M \cong K$ by a linear programming, under a surrogate $l_{1}$ norm minimization of total number of nonzero pixels (irrespective to their values to select only an inhomogeneous solution without the arbitrary mixing homogenous ones). They use purely random unstructured sampling mask of $K$ ones for about $K$ degree of freedoms among all zeros, to make $M$ linear measurements and invert the underdeterminant Mask matrix equation. We follow the convention of electrodes (see V1 to V6 Figure 6).

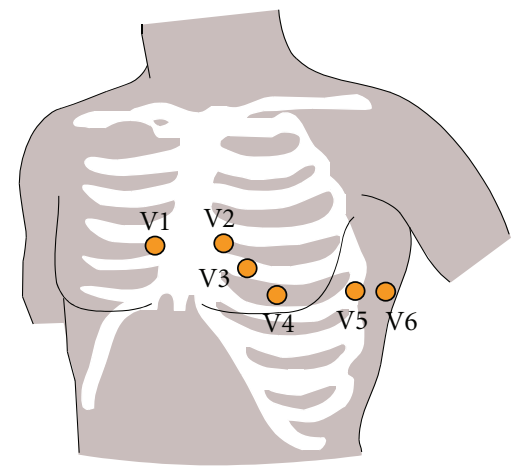

- V1: fourth intercostal space of right of sternum

- V2: fourth intercostal space of left of sternum

- V3: directly between leads V2 and V4

- V4: fifth intercostal space at left midclavicular line

- V5: level with V4 at left anterior axillary line

- V6: level with V5 at left midaxillary line

FIgURE 6: A comfortable halter covers V1 to V6 with nano-grid dry electrodes.

mismatched locations of one's among zeros, which can reduce the number of independent $M$ measurements required up to the degree of sparseness $k$, number of one's $(N \gg m \cong 1.3 k)$ :

$$
\begin{gathered}
R^{N}: \vec{x}=\sum_{n=1}^{N} s_{n} \psi_{n}^{T}=\sum_{n_{k=1}}^{k} s_{n_{k}} \psi_{n_{k}}^{T}=[\Psi] \vec{s}, \\
R^{m}: \vec{y}=\sum_{j=1}^{m} x_{j} \phi_{j}^{T}=[\Phi] \vec{x}=[\Phi][\Psi] \vec{s}=[I C A] \vec{s} .
\end{gathered}
$$

Original data recovery algorithm is a linear program such that

$$
\min .|\vec{x}| \quad \text { s.t. LMS at min. }|\vec{y}-[\Phi] \vec{x}|^{2} \text {. }
$$

Instead of the linear program, we have identified (2) as independent component analysis (ICA) mixing matrix. We can seek several independent killing vectors, bases pursuits, which are orthogonal to all the sparse row vectors except one of the row vectors. A technique has been successfully used in the ill-posed matrix inversion of Independent Component 
TABLE 2: Selected featured ECG commercial devices.

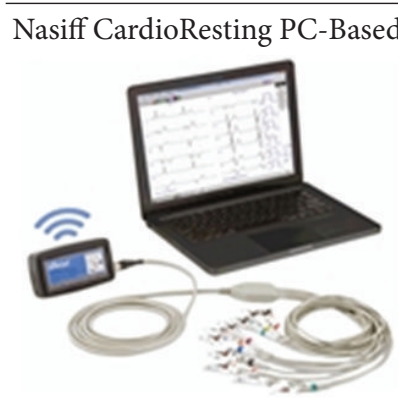

Nasiff Associates, Inc. continues to take the lead in PC-Based Resting ECG systems. Performing fast testing and managing them easily with you at the point-of-care

Tuttnauer 9" Fully Automatic Autoclave

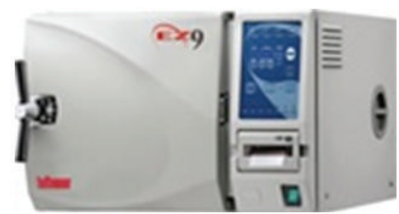

With the simplicity of one-touch design all your sterilization and drying needs are fulfilled. The Tuttnauer EZ9 fully automatic autoclave will fill, sterilize, exhaust, and dry at the touch of a button

CardioCare 2000 Interpretive EKG (PC Connecting Software Included)

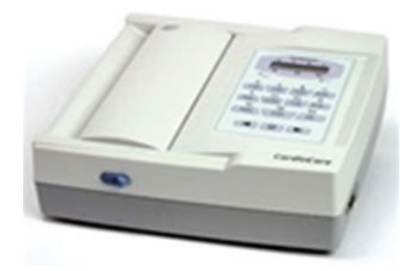

The CardioCare 2000 is an economically priced 12-channel resting ECG that does not compromise on performance. Suitable for use in private practice, ER, or hospital use, the CardioCare 2000 is designed for maximum ease of use and convenience. Software upgradeable and networkable, the CardioCare 2000 is an excellent choice for your diagnostic needs

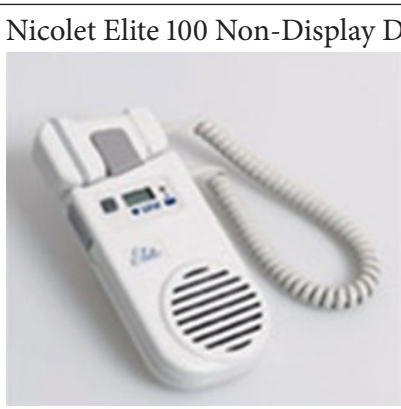

The Elite from Nicolet Vascular offers everything that you need in a Doppler system. The Elite is a configurable, ultrasound Doppler used to detect the fetal heartbeat and to assist in monitoring peripheral arterial and venous blood flow

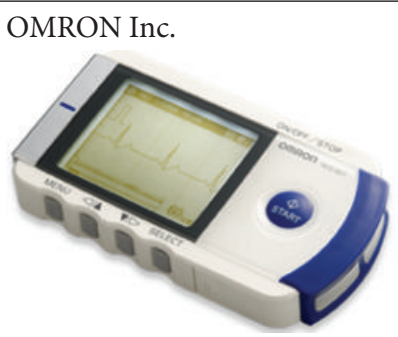

With the OMRON Portable ECG Monitor HCG-801, a recording of about 30 seconds can be made when symptoms occur whether at home or away. These recordings can then be shown to the doctor, who can examine and use the information to assist in correct diagnosis of symptoms

Easy ECG Handheld Monitor FP180

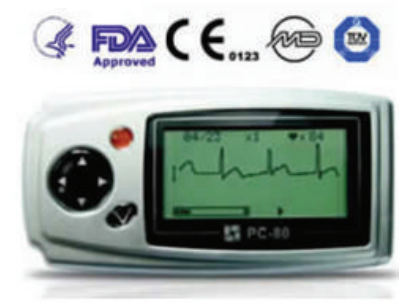

FP180 (PC-80) Easy ECG monitor is a personal single-lead electrocardiographic monitor that records user's cardiac functions and displays the data in a clear and precise waveform for daily health check. This device is intended for self-testing by adult users who might experience transient symptoms that may suggest cardiac conduction abnormity or by adult users whenever they want to have routine checks
Analysis [3]. Our observation is consistent with the mathematics of Compressive Video Sensing, but suggests an organized sparseness video sampling [4]. In other words, these sensory change response vectors have a naturally structured sparseness spanning a pseudoorthogonal subspace that can equally yield a minimum representation of a set of underdetermined linear measurements. Our model may be realized in the working memory of a neuronal synaptic junction network broadcasting a heteroassociative memory (HAM) cross-sensory modalities. The relevant comparison between Artificial Neural Nets/Associative memory (ANN/AM) theory and the CRTD/CS theory comes into the play when to invert an underdetermined set of a short $M$ measurements of data $\vec{y}_{i}$ of the size $i=1,2,3, \ldots M$.

Compressive Sensing: Applying Orthogonal Multiple Input Multiple Output (MIMO) Principle, we monitor ECG's Fourier frequency responses in the following ((i)-(iv)).

(i) term used to describe the breadth of the electrical spectrum viewed by the ECG monitor;

(ii) diagnostic quality is usually $0.05 \mathrm{~Hz}$ to $150 \mathrm{~Hz}$;

(iii) monitor quality is usually $0.5 \mathrm{~Hz}$ to $20-50 \mathrm{~Hz}$;

(iv) usually printed on the ECG recording strip. 


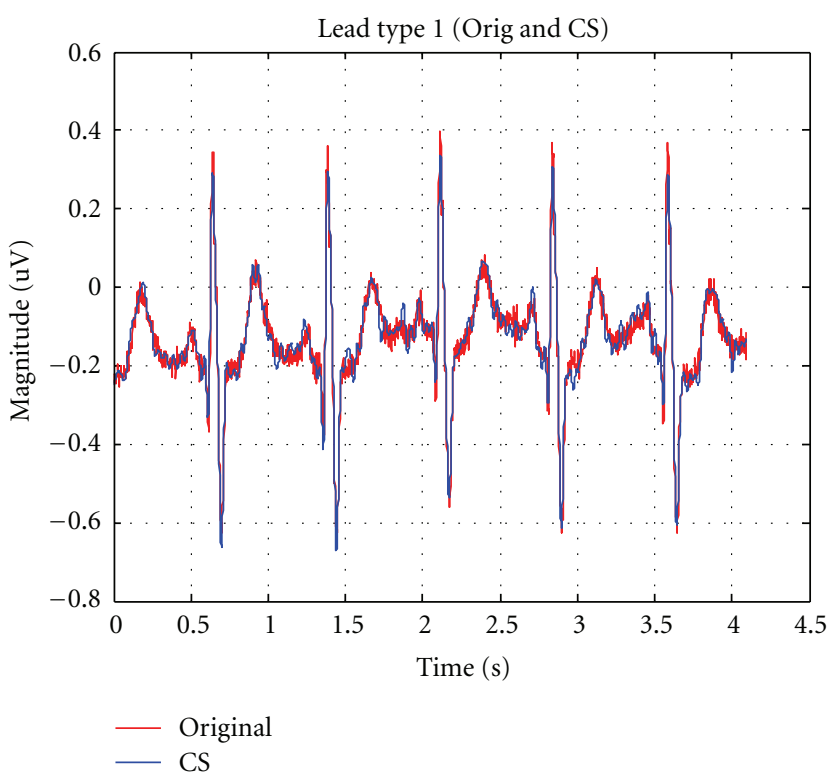

(a)

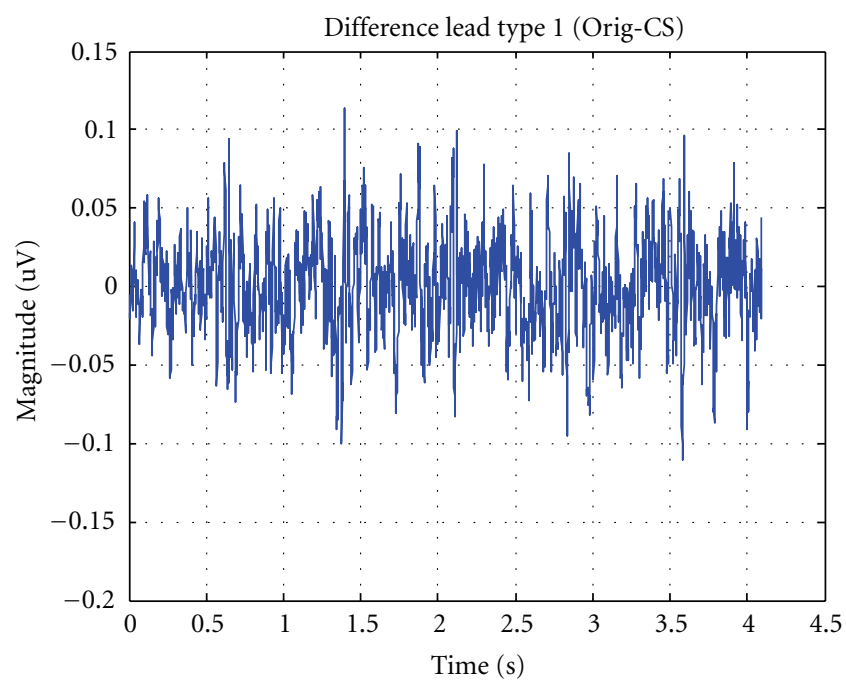

(b)

FIGURE 7: Comparison between the original data and the CS recovering. Figure 7(a) shows lead type 1 at V1, original in blue and the CS in red, where their difference is depicted in Figure 7(b). The difference is less than $0.08 \mathrm{uV}$.

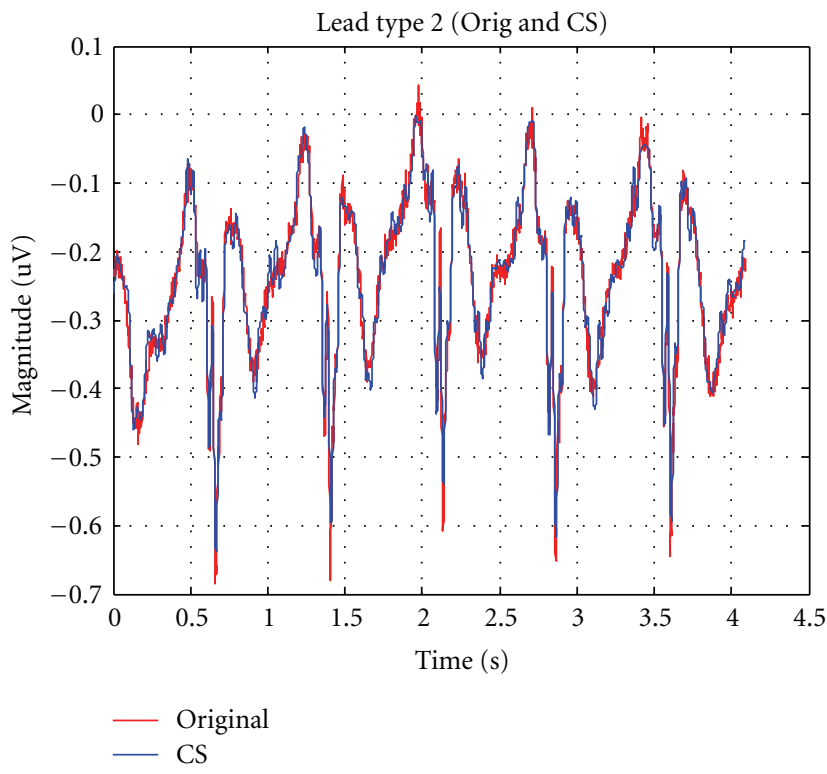

(a)

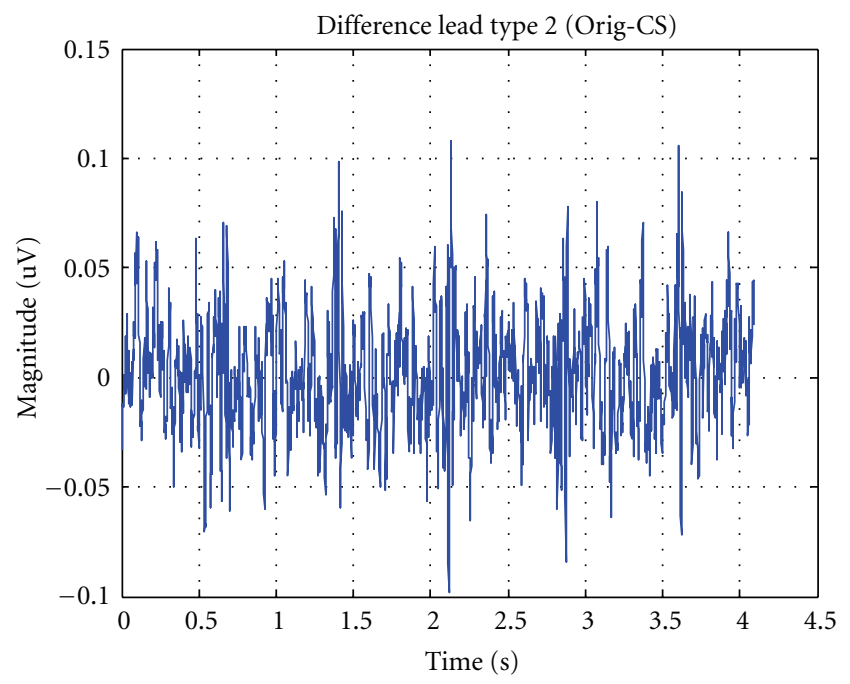

(b)

FIGURE 8: Comparison between the original data and the CS recovering. Figure 8(a) shows lead type 2 at V2, original in blue and the CS in red, where their difference is depicted in Figure 8(a). The difference is less than $0.08 \mathrm{uV}$.

We take Block Sparse Compressive Sensing Code published by Rice University Baranuik et al. 2008 IEEE/IT. The following parameters are used: number of processed samples: 8192; signal sparsity: 16; number of measurements: 1024 FT domain; number of iterations: 20. ECG data resource (http://eleceng.dit.ie/tburke/biomed/assignment1.html).The test results use that the CS algorithm compared to the original ECG data are plotted in Figures 7, 8, 9, 10 and 11 in terms of lead type 1 to 5 , respectively. The original ECG is plotted in red, and the result using CS is plotted in blue. Their difference is depicted on the right.

\section{Conclusion and Additional Modules}

ECG is a relatively noninvasive method to monitoring the heart beats condition, except the tethered wires cable which is somewhat inconvenient during a treadmill stress test, and the cost affordability may be improved for household usages. 


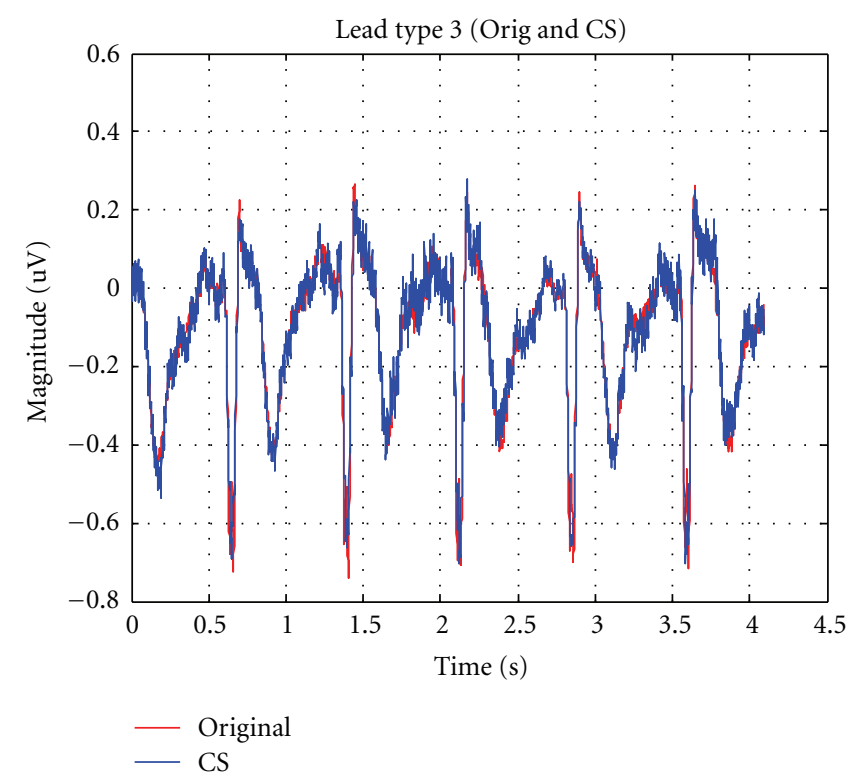

(a)

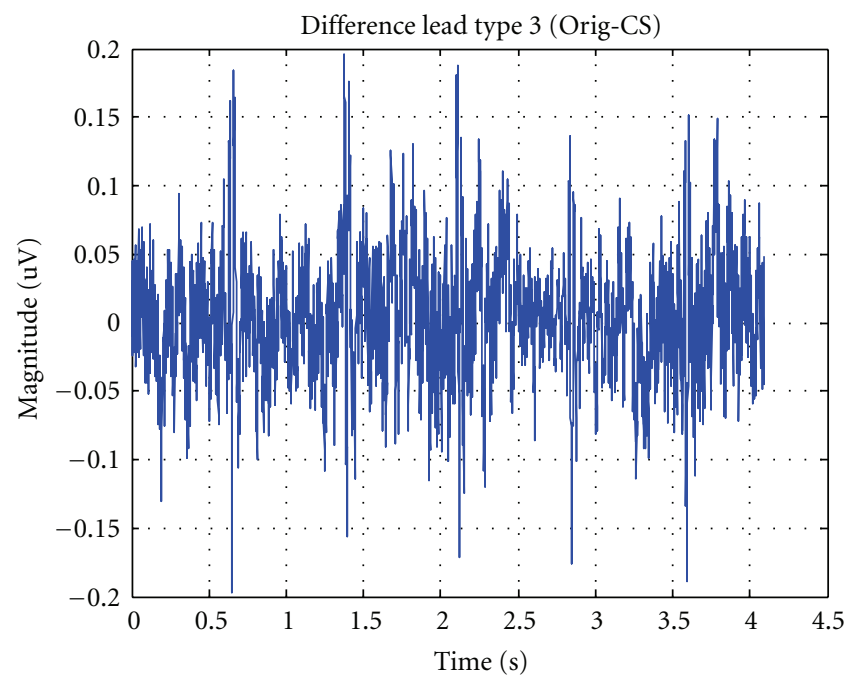

(b)

FIGURE 9: Comparison between the original data and the CS recovering. Figure 9(a) shows Lead type 3 at V3, original in blue and the CS in red, where their difference is depicted in Figure 9(b). The difference is less than $0.08 \mathrm{uV}$.

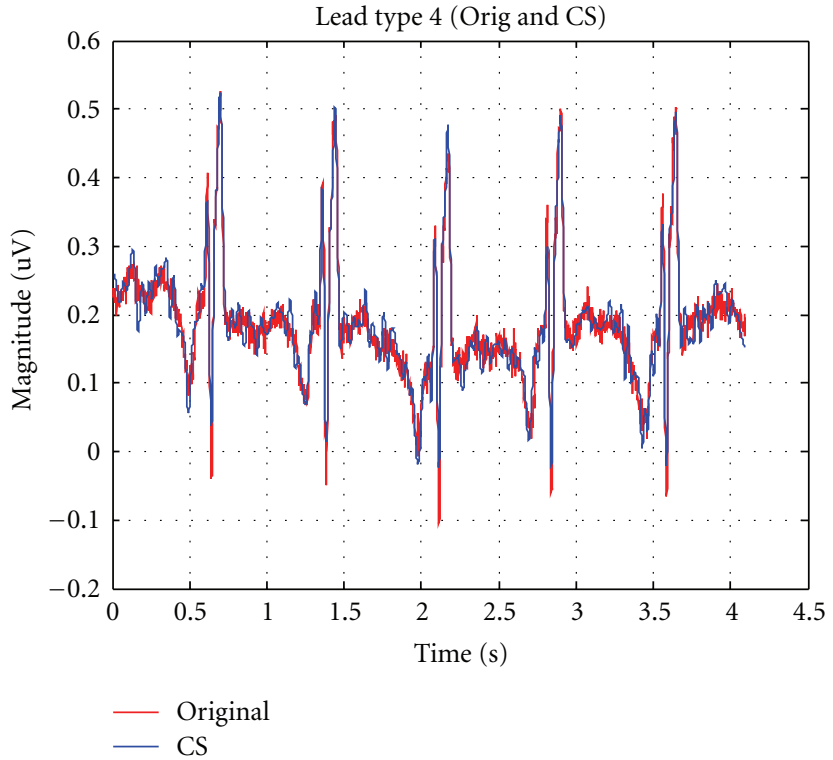

(a)

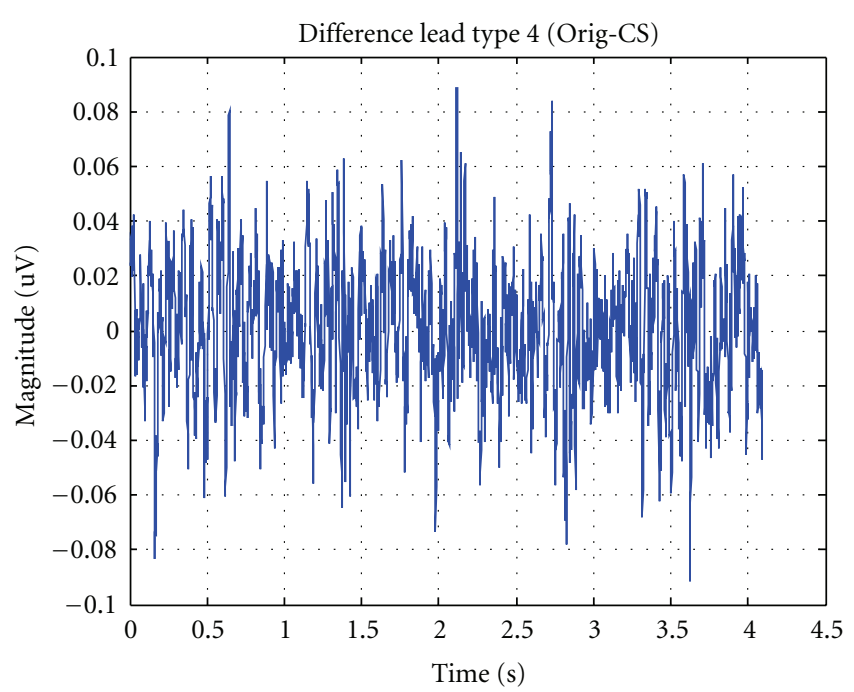

(b)

FIGURE 10: Comparison between the original data and the CS recovering. Figure 10(a) shows lead type 4 at V4, original in blue and the CS in red, where their difference is depicted in Figure 10(b). The difference is less than $0.08 \mathrm{uV}$.

Here we introduce several innovative components towards that popular goal.

The details of other three modules are (i) Learnable Database with management, (ii) Smartphone with computational power for wavelet feature extraction and AiTR, and (iii) Smart Electrodes with mixed signal electronics, which are available in the industrial and the academia community. We will not attempt to summarize them in this limited collaboration. We mentioned in bypassing a smartness material component, related to (iii), in terms of low-power, low impedance, one-directional pull-off nanogrip dry conducting surface (cf. Figure 12(d)), a gecko feet-like. ECG electrodes require usually a sticky pad with lead electrode connector on the pad top. We shall replace them by a readhesive nanonode that is made of an electrical conductor but plastic material. A design has been suggested by Noble Laureate Professor 


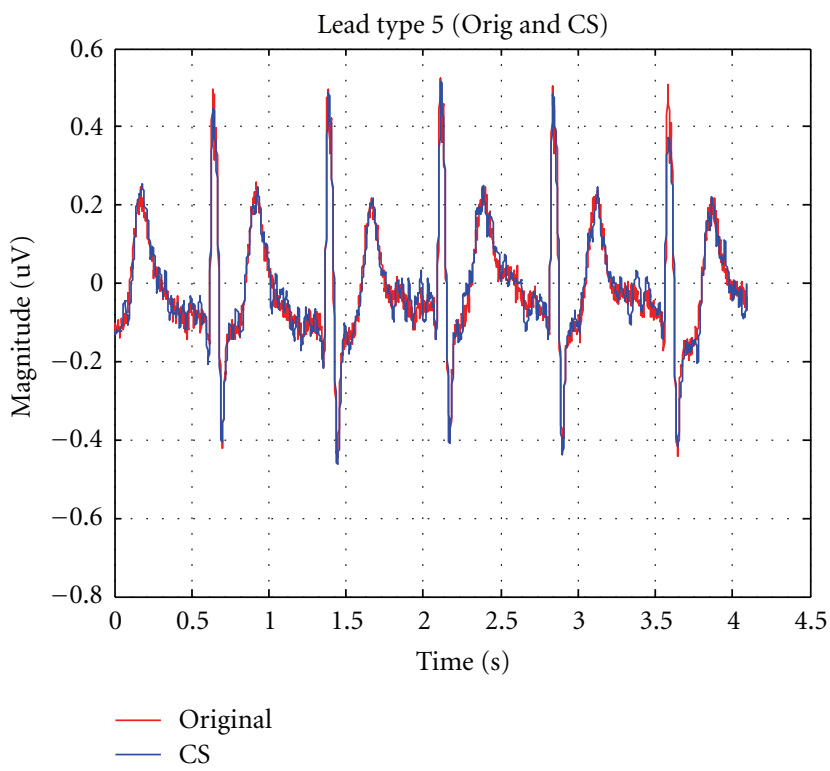

(a)

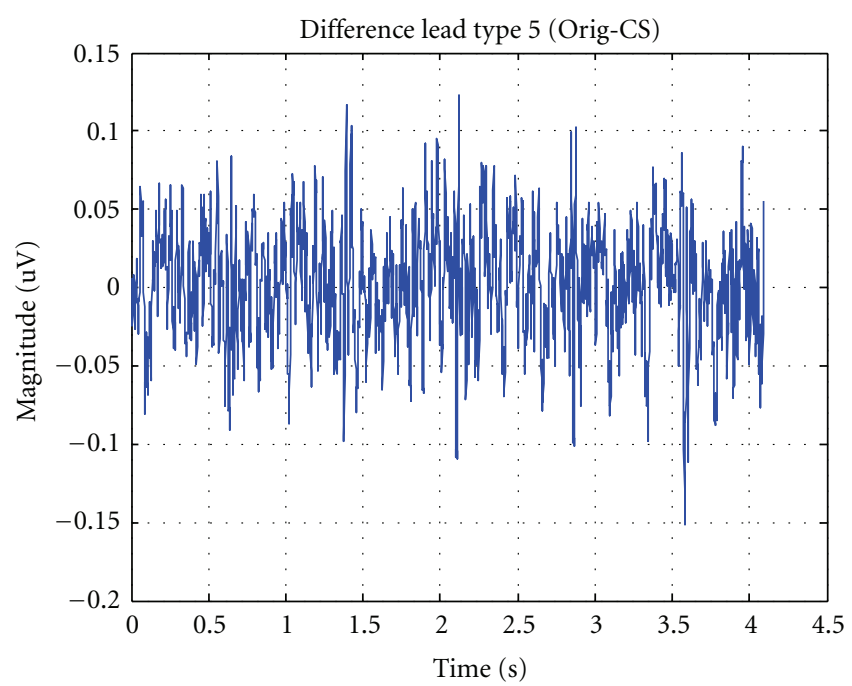

(b)

Figure 11: Lead Type 5 comparison using CS.

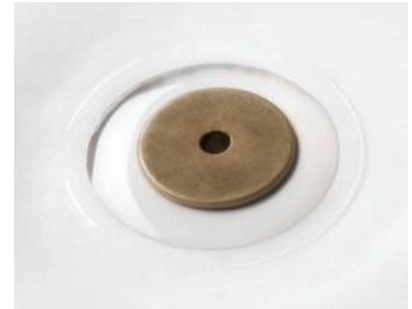

(a)

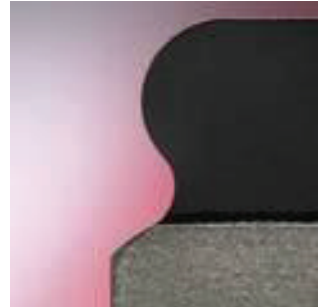

(b)

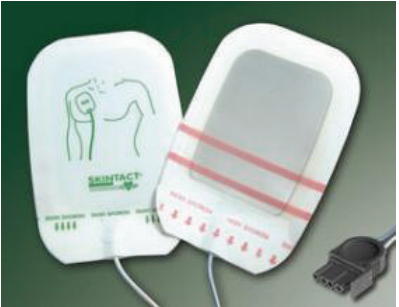

(c)

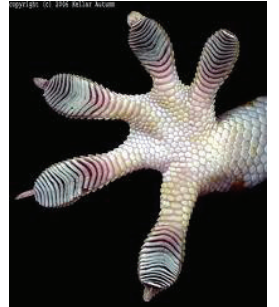

(d)

FIgURE 12: (a) Good electrical contact of ECG electrode made of $\mathrm{Ag} / \mathrm{AgCl}$ sensor; (b) carbon contact interface; (c) commercial pressure sensitive adhesive; (d) a gecko feet.

Grim of Manchester University. Subsequently it was implemented by Defense Advanced Research Program Agency et al. and demonstrated with such a man-made directional grooves material that is used to tailor a glove and shoe for a human to wear them scalping a vertical wall with a much faster speed $\sim 1 \mathrm{~m} / \mathrm{sec}$. The designs of the pressure sensitive adhesive (PSA) feet and treads of the robots were inspired biologically by the toe-peeling mechanism of gecko toes by iRobot Corp [5]. This is likewise adopted for our design of the ECG dry electrodes; wherein Figure 12(b) indicates the possibility of using a traditional bulk carbon material for good ECG contacts. Thus, for example, we suggest a commercial available multiple layers of Carbon Nano Tubes (CNT) in a special direction of the unit crystal axis that becomes a metallic conductor, besides being the semiconductors and the insulators. Then, CNTs are folded in directional loops covering a layer of electrode in a favorable groove pattern having grooved into directional fine inverted groove-like directional nano-hooks of a gecko feet (Figure 12(d)) for "stick and peel" as a reusable good dry electrode.
We can further design new electrode leads which will not suffer of ambient Electromagnetic interference (EMI). Recently, intelligent material has adopted wide-band electric field sensors based on a photonic electric field sensor, as a Macher-Zehnder interferometer, with fibrooptic buried inside a PSA pad. Then, all optics sensors will not suffer of ambient EMI. When the size-weight and power-cost constraints are satisfied, the technology of an electrode less, all optical could be useful for dry electrode design. Meanwhile, NIST at Gaithersburg has demonstrated a miniaturized sugar cube size for optical semiconductor chip magnetometer, which can detect the exceedingly weak magnetic field modulation associated with brain waves and heart beats $[3,6,7]$. The performance is remained to be benchmarked.

\section{References}

[1] H. A. Snellen, Willem Einthoven (1860-1927) Father of Electrocardiography, Life and Work, Ancestors and Contemporaries, Kluwer Academic, Dordrecht, The Netherlands, 1995. 
[2] F. N. Wilson, F. D. Johnston, A. G. Macleod, and P. S. Barker, "Electrocardiograms that represent the potential variations of a single electrode," American Heart Journal, vol. 9, no. 4, pp. 447458, 1934.

[3] T. H. . Sander, J. Preusser, R. Mhaskar, J. Kitching, L. Trahms, and S. Knappe, "Magnetoencephalography with a chip-scale atomic magnetometer," Biomedical Optics Express, vol. 3, no. 5, pp. 981-990, 2012.

[4] H. Szu, C. Hsu, J. Jenkins, J. Willey, and J. Landa, "Capture significant events with neural networks," Neural Networks, vol. 29-30, pp. 1-7, 2012.

[5] K. . Autumn, S. T. Hsieh, D. M. Dudek, J. Chen, C. Chitaphan, and R. J. Full, "Dynamics of geckos running vertically," The Journal of Experimental Biology, vol. 209, no. 2, pp. 260-272, 2006.

[6] S. Knappe, T. H. Sander, O. Kosch, F. Wiekhorst, J. Kitching, and L. Trahms, "Cross-validation of microfabricated atomic magnetometers with superconducting quantum interference devices for biomagnetic applications," Applied Physics Letters, vol. 97, no. 13, Article ID 133703, 2010.

[7] W. C. Griffith, S. Knappe, and J. Kitching, "Femtotesla atomic magnetometry in a microfabricated vapor cell," Optics Express, vol. 18, no. 26, pp. 27167-27172, 2010. 

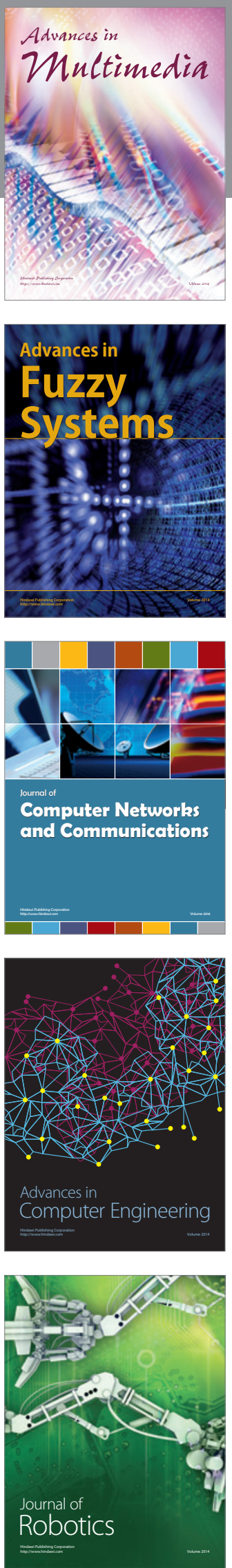

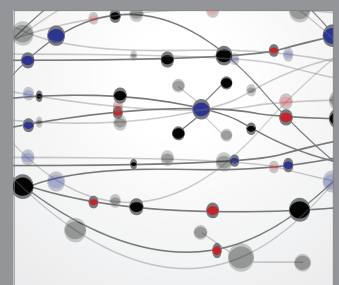

The Scientific World Journal
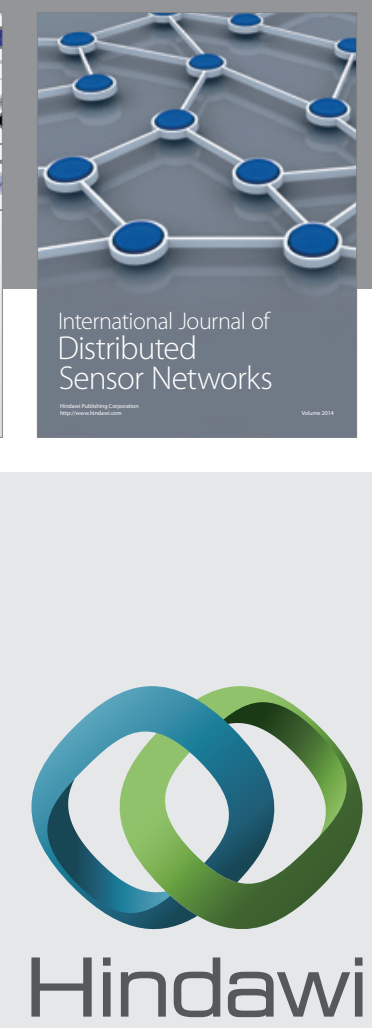

Submit your manuscripts at

http://www.hindawi.com
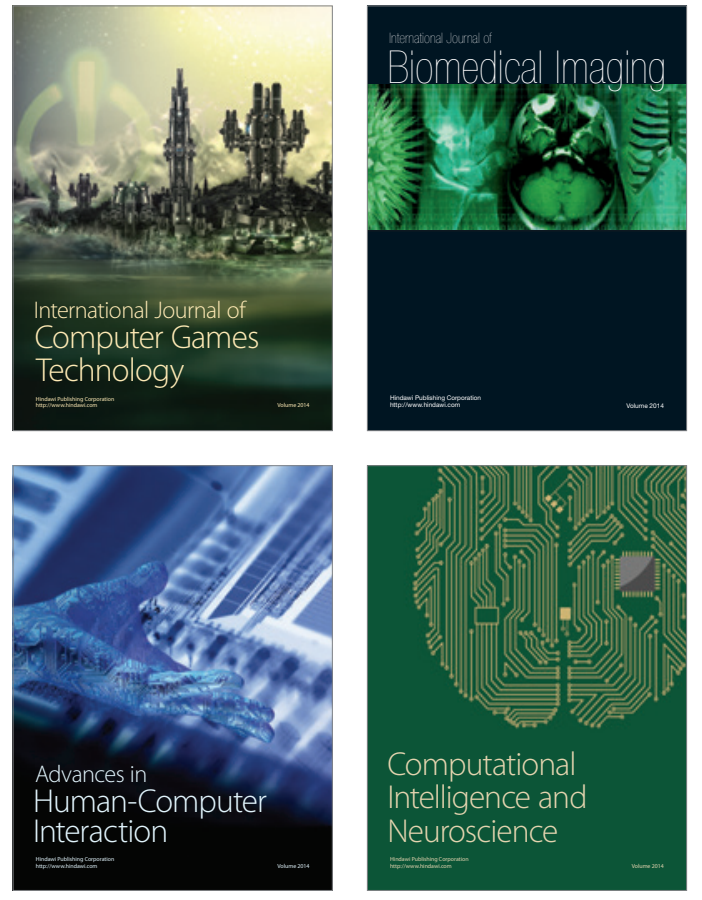
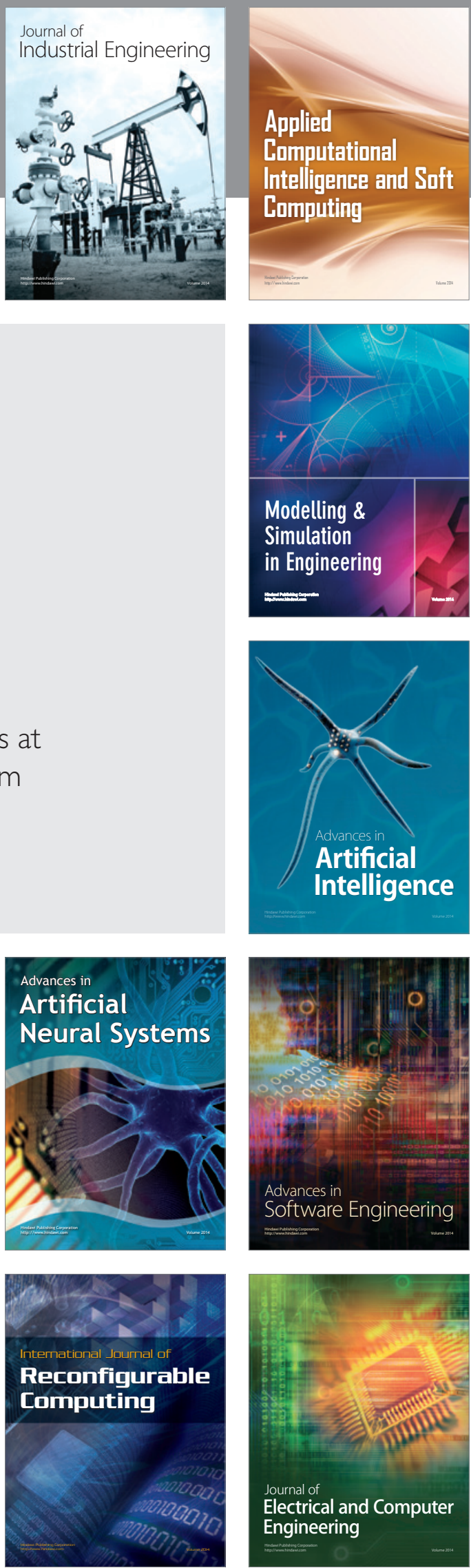